\title{
Forecasting the deterioration of cement-based mixtures under sulfuric acid attack using support vector regression based on Bayesian optimization
}

\author{
Linping $\mathrm{Wu}^{1} \cdot$ Chaoshi $\mathrm{Hu}^{2} \cdot$ Wei Victor $\mathrm{Liu}^{1}$ (i)
}

Received: 27 February 2020 / Accepted: 28 October 2020 / Published online: 9 November 2020

(c) Springer Nature Switzerland AG 2020

\begin{abstract}
Immersion test is time-consuming and labor-intensive in evaluating the resistance of concrete against sulfuric acid attack. An alternative way for acid resistance evaluation is to predict the sample deterioration through machine learning methods using a currently available database. However, current predictive models have failed to include testing conditions, which limits their applications to certain testing conditions. Accordingly, predictive models need to be developed to include parameters of both mixture design and testing conditions in the forecasting of deterioration of mortar under sulfuric acid attack. In this study, such predictive models were established using the Bayesian optimized-support vector regression (BO-SVR) algorithm. Prediction errors were calculated, and a superiority test was conducted to evaluate the performance of the proposed BO-SVR models. It was found that the proposed BO-SVR model outperformed the other models in predicting the mass change and the compressive strength. This provides a new way of evaluating the acid resistance of cement-based materials.
\end{abstract}

Keywords Support vector regression · Prediction · Testing conditions · Acid resistance $\cdot$ Mortar

\begin{tabular}{|c|c|c|c|}
\hline \multicolumn{2}{|c|}{ List of symbols } & \multirow[t]{2}{*}{$N_{F}$} & \multirow{2}{*}{$\begin{array}{l}\text { The number of parameters used for perfor- } \\
\text { mance evaluation }\end{array}$} \\
\hline$A A E$ & Average absolute error & & \\
\hline \multirow[t]{2}{*}{$A_{C V}$} & Value of the performance indicators for the & $n$ & Number of samples \\
\hline & $k$-fold cross-validation & $n_{1}$ & Number of input parameters \\
\hline$b$ & Offset scalar of SVR function & $P_{i}$ & Value of performance indicator in each data \\
\hline C & Penalty factor & & fold \\
\hline$E P R$ & Evolutionary polynomial regression & $R^{2}$ & Coefficient of determination \\
\hline$f(x)$ & SVR predicted value & $R_{F}$ & The sum of the average rank of performance \\
\hline$F M$ & Test statistic of Friedman's test & & parameters for each model \\
\hline$K\left(x_{i}, x_{j}\right)$ & Kernel function & $s_{i}$ & The rank of $\mathrm{i}^{\text {th }}$ parameter for performance \\
\hline$k$ & Number of data fold & & evaluation for each model \\
\hline$k_{F}$ & Number of models for comparison & $w$ & SVR function slope \\
\hline$\Delta m$ & Mass change & $x_{i}$ & Input vector in the training dataset \\
\hline \multirow{2}{*}{$m_{n}$} & Mass of sample after a certain period of & $x^{\prime}$ & Standardized data \\
\hline & immersion & $x_{\min }$ & Minimum value of the input parameter \\
\hline$m_{0}$ & Mass of sample before immersion & $x_{\max }$ & Maximum value of the input parameter \\
\hline$N$ & Number of neurons in the hidden layer & $y_{i}$ & Output value in the training dataset \\
\hline
\end{tabular}

$\triangle$ Wei Victor Liu, victor.liu@ualberta.ca | ${ }^{1}$ Department of Civil and Environmental Engineering, University of Alberta, Edmonton, Alberta T6G 2E3, Canada. ${ }^{2}$ Utility Planning \& Design, EPCOR Utilities Inc, Edmonton, Alberta T5M 3B8, Canada. 


$\begin{array}{ll}y_{i}^{\prime} & \text { Predicted value } \\ a_{i} \alpha_{i}^{*} & \text { Lagrange multipliers } \\ \epsilon & \text { Tolerable error } \\ \xi_{i}, \xi_{i}^{*} & \text { Slack variables } \\ \sigma & \text { Width of radial basis function }\end{array}$

\section{Introduction}

The sulfuric acid attack poses serious problems for cementbased sewer tunnels: a significant service life reduction to the structures and a substantial financial cost for repair [1]. To mitigate this sulfuric acid attack, much research has been carried out in seeking a more acid-resistant cementbased mixture. For example, previous studies have proven that the addition of pozzolanic materials, including fly ash $[2,3]$, silica fume $[4,5]$, and metakaolin $[1,6]$ can slow the deterioration by sulfuric acid attack. A large amount of data has been published regarding the deterioration of these mixtures exposed to sulfuric acid attack. However, these data are not comparable due to the difference in testing conditions in various studies. Thus, a new evaluation test is often carried out when a comparison of these mixtures is needed, which is time-consuming and labor-intensive. The immersion test often lasts for several months or even several years. Herein, many researchers have been trying to find alternative ways to evaluate acid resistance. One of the options is to predict the deterioration of concrete by empirical models or regression learning algorithm using a currently available database.

The first way to predict deterioration is to develop empirical equations for the prediction of the deterioration of mixtures under sulfuric acid attack. De Belie et al. [7] developed an equation to predict the degradation depth under sulfuric acid attack using experimental data, considering the alkalinity and water absorption of the mixtures. A correlation coefficient of 0.84 was found between input parameters and the corrosion depth. More studies have been done to develop empirical models for the deterioration prediction of samples subjected to sulfate attack [8-10]. However, these empirical models only included parameters about mixtures and ignored the effects of testings conditions on the deteriorations. This limits the application of current models to certain testing conditions. For instance, the empirical model published by Kurtis et al. [8] was developed to predict the deterioration of concrete specimens soaked in $2.1 \%$ sodium sulfate solution for 40 years, while the application of the model proposed by Lee et al. [10] was limited to concrete specimens exposed to $10 \%$ sodium sulfate solution and $10 \%$ magnesium sulfate solution for one year. When the testing conditions are changed, the accuracy of these models is unknown.
Another alternative way to forecast deterioration is through a machine learning algorithm such as artificial neural networks (ANN) and evolutionary polynomial regression (EPR). Using the data collected from their experimental tests, Hewayde et al. [11] developed an ANN model for the prediction of compressive strength and mass change of 78 mixtures under a sulfuric acid attack. The ANN models showed reasonable accuracy in the prediction with an average absolute error (AAE) of $8.45 \%$ for mass change prediction and $4.85 \%$ for compressive strength prediction. Using the same database as Hewayde et al. [11], Alani et al. [12] proposed an EPR method to forecast the mass loss of concrete subject to sulfuric acid attack. Slightly higher accuracy was reported for the EPR method compared with the ANN model in mass change prediction. However, these models also failed to include the testing conditions in their studies. Only mixture design parameters were used as inputs in these two models.

Much research has reported that the testing conditions (e.g., acid concentration, reaction surface area, continuous wetting-drying cycles, immersion time, and surface area to acid volume ratio) could have a significant influence on concrete deterioration [13-15]. For example, Chen et al. [16] found that immersion time is one of the main factors affecting the compressive strength of concrete after immersion. Through experimental observation, Attiogbe et al. [15] found that wetting-drying cycles increased the concrete deterioration degree. Romben et al. [13] stated that any testing conditions that would affect the acid supply would influence the deterioration of concrete. Herein, a prediction without considering the testing conditions could limit the application of the proposed model to certain testing conditions. Thus, there is an urgent need to develop a predictive model that incorporates these testing conditions.

With the rapid development of machine learning techniques, there are various algorithms available for the construction of such predictive models, such as ANN and EPR, mentioned in the previous review. However, these ANN and EPR models have their shortcomings. For instance, ANN has a complex network structure with matrixes of weights and biases, which demands expensive computational time and memory $[17,18]$. In addition, due to a large number of weights, the ANNs are prone to overfitting, which leads to poor generalization $[19,20]$. For the EPR methods, they were found to be too sensitive to outliers in a database. A single outlier in data could lead to a significant error in the prediction results [21]. Thus, a model is needed to overcome these shortcomings.

In this study, the main aim is to develop a predictive model to forecast deteriorations of mortars under sulfuric acid attack incorporating both mixture design parameters and test conditions. Two Bayesian optimization-support 
vector regression (BO-SVR) models were constructed to predict mass change (model I) and residual compressive strength (model II) of mortar samples under a sulfuric acid attack. The SVR algorithm was adopted because it was found to have a lower risk of overfitting [22], and relatively low sensitivity to outliers due to the use of loss functions [23]. Furthermore, SVR models have excellent ability to search for global optimal solutions with a relatively small database [24]. This applies to the database size in this study (244 data points for mass change prediction and 93 data points for strength prediction). Lastly, the SVR models are easy to construct because SVR models have fewer model variables $[25,26]$. The BO technique was adopted to search the optimal hyperparameters for the SVR model because of its high accuracy and efficiency [27]. The input parameters included both mixture designs and testing conditions. The performance of the BO-SVR models was evaluated by prediction errors and a superiority test. This study has provided valuable insights into the deterioration prediction of mortar samples under different sulfuric acid immersion conditions.

The rest of this paper is structured as follows. Section 2 provides details of the proposed BO-SVR model, including the methods to evaluate its performance and superiority. Section 3 explains the development of the database for predictive modeling. Section 4 discusses the performance of the proposed BO-SVR model in predicting mass change and compressive strength. Finally, Sect. 5 summarizes the main findings of this study.

\section{Methodology}

In this section, an SVR model optimized by the Bayesian algorithm was proposed to predict the mass change and compressive strength of mortar samples under a sulfuric acid attack. To assess the performance of the BO-SVR model, five other models were constructed for comparison, including SVR models optimized by grid search (GS), random search (RS), and particle swarm optimization (PSO), a twin SVR (TSVR) model and a traditional ANN model. All the models were verified using the $k$-fold crossvalidation technique. The performance of each model was evaluated by four averaged indicators: the coefficient of determination $R^{2}$, the mean absolute error (MAE), the root mean square error (RMSE), and the mean absolute percent error (MAPE). The overall methodology is illustrated in Fig. 1.

\subsection{Support vector regression}

The SVR algorithm [28] was adopted in this study. The basic idea of SVR is to nonlinearly map the training dataset (input data and output data) into a high-dimensional feature space via a kernel function. A linear relationship between input data and output data can be found in this high-dimensional feature space to reflect the nonlinear relationship before mapping. This linear function in the high-dimensional space is the SVR function. Supposing $T=\left\{\left(x_{1}, y_{1}\right),\left(x_{2}, y_{2}\right), \ldots,\left(x_{n}, y_{n}\right)\right\}$ is a training data set, where $n$ is the sample number, $x_{i} \in R^{n}$ is the input vector, and $y_{i} \in R$ is the corresponding output. The SVR function can be written as follows:

$f(x)=w^{T} x+b$

where $f(x)$ is the predicted value, $w \in R^{n}$ indicates the slope of the function, and $b \in R$ is an offset scalar.

By introducing a tolerance $\epsilon$ and slack variables $\xi_{i}, \xi_{i}^{*}$, the SVR function can be written as an optimization problem:

$\operatorname{minimize} \quad \frac{1}{2} w^{T} w+C \sum_{i=1}^{i=n}\left(\xi_{i}+\xi_{i}^{*}\right)$

s.t. $\quad y_{i}-f\left(x_{i}\right) \leq \epsilon+\xi_{i}$

$f\left(x_{i}\right)-y_{i} \leq \epsilon+\xi_{i}^{*}$

$\xi_{i}, \xi_{i}^{*} \geq 0$

In this optimization problem, an error of $\epsilon$ is allowed for each datapoint. In other words, a predicted $f\left(x_{i}\right)$ with an error smaller than $\epsilon$ means $f\left(x_{i}\right)$ is considered a correct prediction, and no penalty will be applied to the objective function. When an error is larger than $\epsilon$, the extra error will be captured by the slack variables $\xi_{i}, \xi_{i}^{*}$ and penalized in the objective function through a penalty factor $C$.

To solve this optimization problem, the optimization problem is often transformed into its dual form where the constraints are expressed by Lagrange multipliers [29]:

$\operatorname{minimize} \frac{1}{2} \sum_{i=1}^{i=n} \sum_{j=1}^{n}\left(\alpha_{i}-\alpha_{i}^{*}\right)\left(\alpha_{j}-\alpha_{j}^{*}\right) x_{i}^{T} x_{j}$

$$
+\epsilon \sum_{i=1}^{n}\left(\alpha_{i}+\alpha_{i}^{*}\right)-\sum_{i=1}^{n} y_{i}\left(\alpha_{i}-\alpha_{i}^{*}\right)
$$

s.t. $\sum_{i=1}^{n}\left(\alpha_{i}+\alpha_{i}^{*}\right)=0$;

$0 \leq \alpha_{i}, \alpha_{i}^{*} \leq C$ 


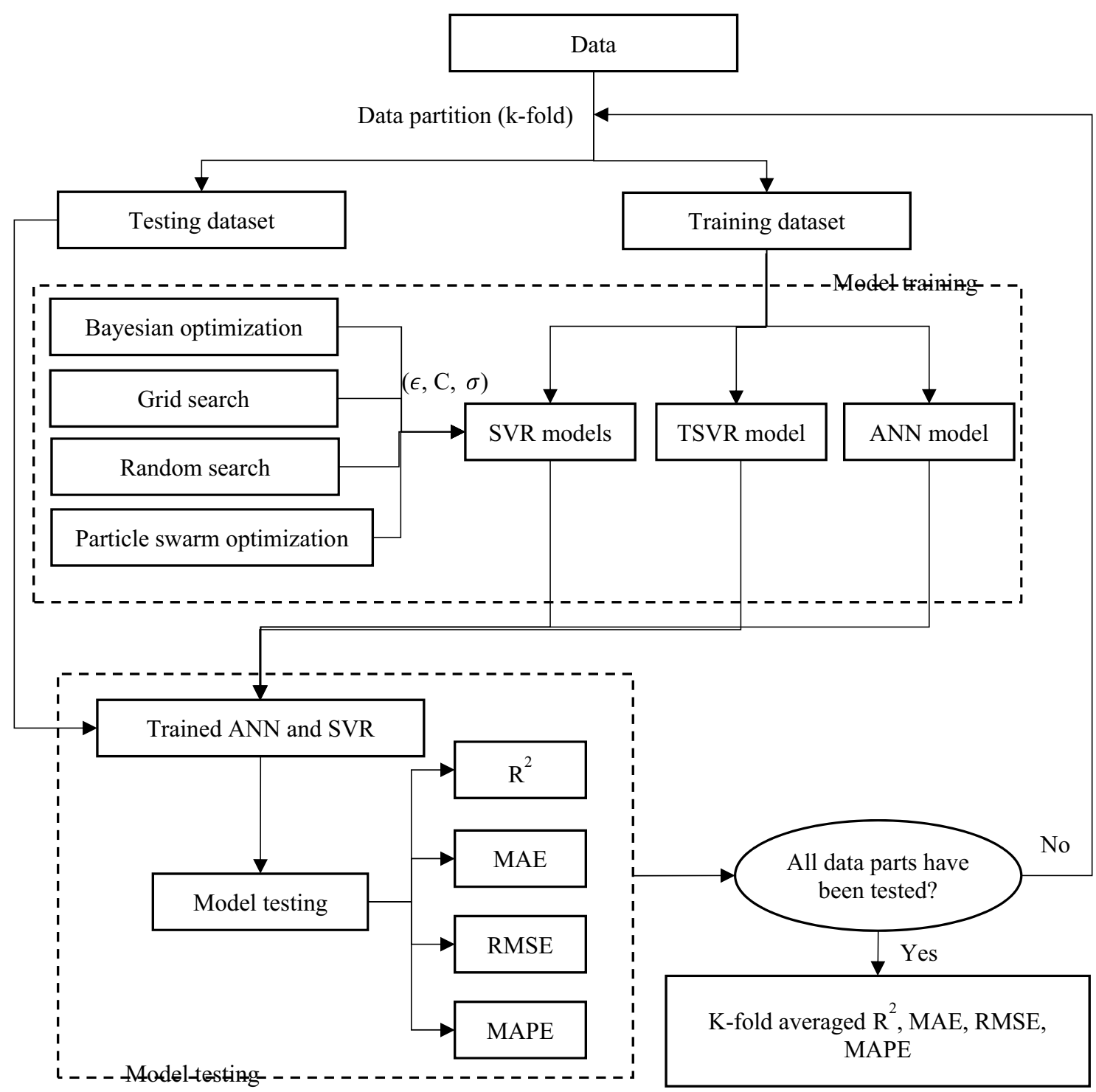

Fig. 1 A flowchart showing the overall methodology

And the SVR function can be rewritten as follows:

$f(x)=\sum_{i=1}^{n}\left(\alpha_{i}^{*}-\alpha_{i}\right) K\left(x_{i}, x\right)+b$

where $\alpha_{i}$ and $\alpha_{i}^{*}$ are Lagrange multipliers. The support vector is the training vector with non-zero Lagrange multipliers. The dot product $x_{i} x$ is the kernel function $K\left(x_{i}, x_{j}\right)$.

Typically, there are three kernel functions available for the SVR modeling including radial basis function (RBF), polynomial kernel function, and sigmoid function. Since the RBF kernel function is capable of solving nonlinear relationship problems and was reported to perform better than polynomial and sigmoid function $[30,31]$, the
RBF kernel $K(x, y)=\exp \left(-\left(x-x_{i}\right)^{2} / 2 \sigma^{2}\right)$ is adopted in this research. Variable $\sigma$ is the width of the kernel function.

\subsection{Implementation of optimization in SVR models}

The performance of nonlinear SVR is highly dependent on the selection of hyperparameters (e.g., $\epsilon, C$, and $\sigma$ for RBF-based SVR) [32-34]. Careful tuning of these parameters is essential for improving the performance of the SVR model. Typical methods of hyperparameters optimization include GS, RS, and PSO. GS is to try every possible combination of hyperparameters until the stop criteria is met [35]. The stop criteria could be that the pre-set max iteration step is reached, or all the possible combinations of hyperparameters have been tried. The combination of 
hyperparameters with the lowest objective function is then chosen as the hyperparameters for the SVR model. However, as this method searches all possible combinations of hyperparameters, it requires large computational time and memory [36]. RS is to find the optimal combination of hyperparameters by randomly choosing possible values of hyperparameters [37]. RS is more efficient than GS in seeking hyperparameters as only part of the possible solutions is tried. However, the RS has a major drawback of high variance resulting from random trials of solutions. This makes the method difficult in reproducing results [36]. In PSO, positions and velocities are randomly assigned to a set of particles to seek the hyperparameters with the lowest objective function [38]. It utilizes information from previous iterations to update the positions and velocities of particles, which makes it efficient in hyperparameter optimization. However, it was found that PSO tends to provide local optimum and has a low convergence rate [39]. Compared with these methods, the BO technique was reported to provide higher accuracy and efficiency in finding the global optimal hyperparameters [27]. Thus, this research adopted the BO approach to find the optimal hyperparameters for the SVR models. The SVR models optimized with GS, RS and PSO were also established as the references to evaluate the performance of $\mathrm{BO}$ algorithm in hyperparameter optimization.

The implementation of BO-SVR model is presented in Fig. 2 and described as follows:

Step 1: Data preparation: the input data in the dataset were first normalized to $[0,1]$. Then, the dataset was randomized and divided into five parts for the k-fold cross-validation of the model. When dividing the dataset into a training dataset and a testing dataset, random sampling may result in biased consequences [40]. It is likely that the high prediction accuracy of the randomly divided test dataset $A$ cannot be reproduced from another randomly selected test dataset $B$. Thus, to minimize this bias, the $k$-fold cross-validation technique was employed in this study. The dataset was randomly divided into five parts; each part has the same propor-
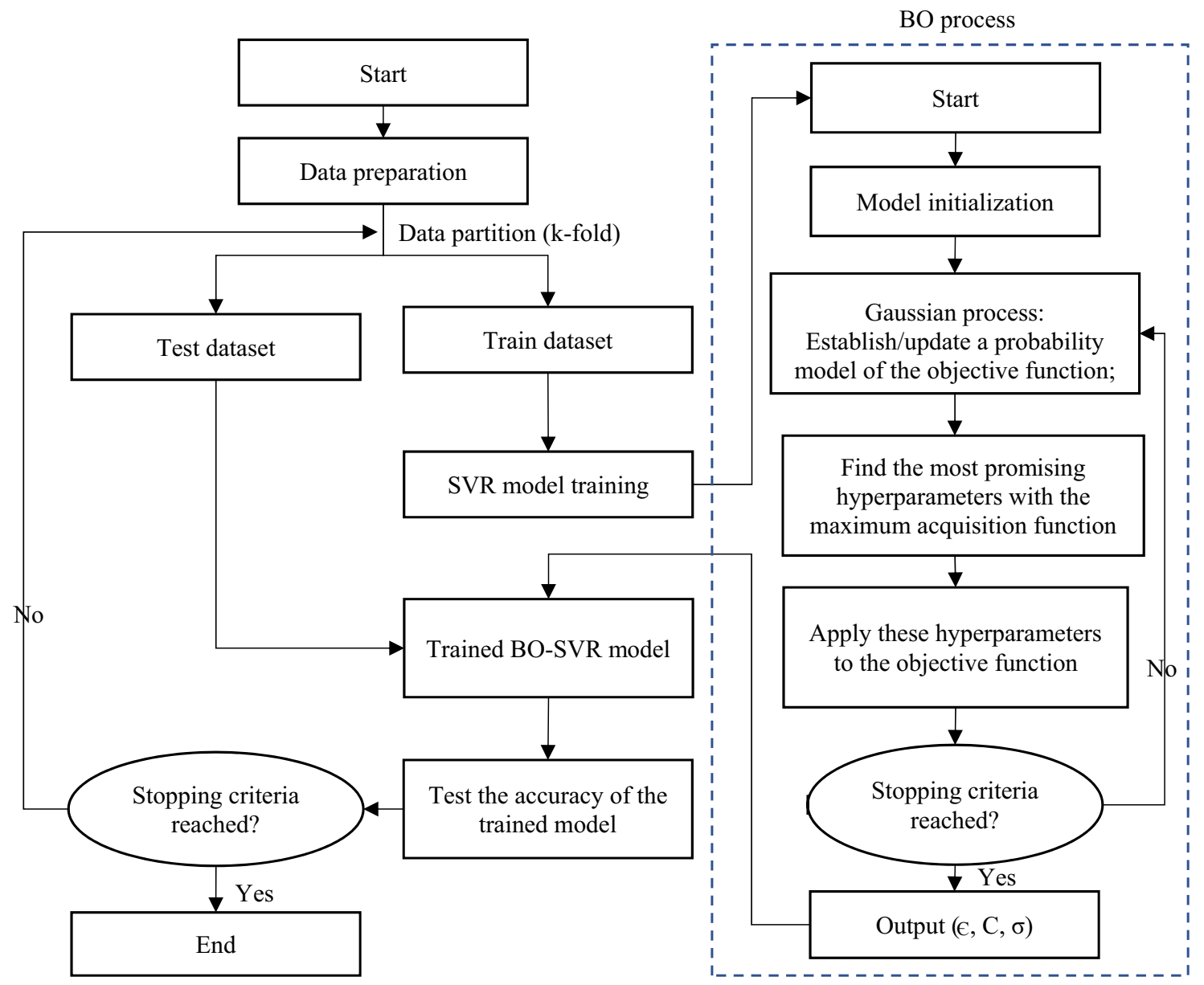

Fig. 2 The flowchart of BO-SVR modeling 
tion of the total dataset. The model was run five times until all five parts of data had been used as a testing dataset.

Step 2: Model training: during the training process, the BO approach was adopted. In the BO process, a probabilistic distribution (statistical model) of the objective function is first constructed through a Gaussian process. This probabilistic distribution describes the potential values of the objective function at different points. In this study, the objective function was to minimize the mean square error (MSE) between the observed values with the predicted values:

MSE $=\frac{1}{n} \sum_{i=1}^{n}\left(y_{i}-y_{i}^{\prime}\right)^{2}$

where $n$ is the number of the forecasting points, and $y_{i}$ and $y_{i}^{\prime}$ are the actual and predicted values, respectively.

Then, a combination of hyperparameters is selected based on an acquisition function. The acquisition function measures how much improvement will be made on the model to try a point (a combination of hyperparameters). The point with the highest expected improvement is picked as the next sample point. These selected hyperparameters are then applied to the objective function, and the probabilistic distribution is updated, incorporating the new results. These steps are repeated until the stop criteria are met. At last, the output is the optimized combination of hyperparameters that will be used in the trained SVR model. Bayesian optimization makes full use of all the information from previous iterations to find the next data point. Herein, the $\mathrm{BO}$ is able to find the global optimal solution with relatively fewer iterations [41, 42].

The abovementioned training process was carried out in Matlab 2019a environment on a personal computer (PC) with an Intel Core i5-6200 (2.3 GHz) processor having 8GB of memory. The maximum iteration steps were set to 100 for mass change prediction and compressive strength prediction after running several trials.

Step 3: Model testing - test the model performance with the testing dataset. This will be discussed in Sect. 2.4.

Step 4: Use the next part of data as the testing dataset, and repeat steps 2 and 3 until every part has been used as the testing data.

The implementation of GS-SVR, RS-SVR and PSO-SVR followed similar steps described above except some differences in step 2. For GS, every possible combination of hyperparameters will be tried until the max iteration is reached. In order that a sufficient range of hyperparameters can be searched, the max iteration was set to a large number of 1000 for the GS-SVR model. For RS, the max iteration was set as 100 based on the results from several trials. The implementation of PSO-SVR followed a similar procedure described as a relevant study by Meng et al. [38]. The random positions and velocities are first assigned to particles, and then the training dataset is used to train the model and find the best particle positions with the lowest objective function. After that, the particle velocities and positions are updated for the next round of model training. The procedure is repeated until the max iteration step (200) is reached. The position with the lowest objective function will be adopted as the combination of hyperparameters for the model.

In addition to the previous SVR models, a twin SVR (TSVR) model was established as a comparison. The TSVR algorithm was first proposed by Peng [43] in 2010 and has become a popular method due to its computational simplicity [44]. Its popularity has made it a good reference for evaluating other models. The basic idea of the TSVR is to generate two functions. Each function determines the down-bound or up-bound of the regressor. In this way, the TSVR solves two small quadratic programming problems rather than solving a large one in the traditional SVR. In this study, a TSVR model was established following a similar procedure in the study by Shao et al. [45]. A successive overrelaxation (SOR) technique was adopted to solve the optimization problems.

\subsection{Development of artificial neural networks}

To provide a performance comparison, an ANN model was also constructed. Due to its strong ability to model complex and nonlinear relationships, ANN is one of the most commonly used machine learning methods for prediction problems of cement-based materials [46]. This makes ANN a good reference in evaluating the performance of the BO-SVR model. ANN is a computational model that can mimic biological neural networks [47]. It consists of three layers: the input layer, the hidden layer, and the output layer. The input layer receives the value of input factors that influence the outputs [48]. This layer has no activities during the computational process. The hidden layer adds non-linearity to the system via an activation function, and it carries the weights and biases describing the strength of the connection between linked neurons [49]. The output layer contains the ANN prediction values. During the training process, the weights and biases will be updated through backpropagation to minimize the error between the predicted results and the actual values.

To construct an ANN model, the selection of neuron number in the hidden layer is of great significance. More neurons in the hidden layer can reduce the error of the model but can also result in overfitting of the model [11]. If the number of neurons is small, underfitting may occur. 
In this study, the number of neurons was determined via Eq. (6) below, which was proposed by Sheela et al. [50], because it produced the lowest MSE compared with other methods. In order to avoid overfitting, Bayesian regularization was adopted in this research due to its superior generalization capability [51].

$N=\frac{\left(4 n_{1}^{2}+3\right)}{\left(n_{1}^{2}-8\right)}$

where $N$ is the number of neurons in the hidden layer; $n_{1}$ denotes the number of input parameters.

Figure 3 shows the structures of ANN models for mass change and compressive strength predictions. For mass change prediction, there were 11 input parameters (6 about mixture design, and 5 about testing conditions), 5 neurons in the hidden layer, and one output parameter (mass change), while the ANN model for compressive strength prediction contained 12 input parameters (7 about mixture design/properties, and 5 about testing conditions), 5 neurons in the hidden layer, and one output parameter (compressive strength). The $k$-fold cross-validation technique was also adopted in the ANN modeling.

\subsection{Performance criteria}

To assess the optimization performance of the BO technique, SVR models with three other optimization techniques were also constructed. These techniques include GS, RS, and PSO [38]. The techniques were chosen because they have been frequently used as the optimization tools in machine learning and have provided good solutions in searching the hyperparameters $[36,52,53]$. This makes these optimization techniques good references. In total, six predictive models were constructed. To evaluate the performance of these predictive models, four performance indicators were calculated in this study, including the $R^{2}$, MAE, RMSE, and MAPE, which can be calculated as Eqs. (7), (8), (9), (10). The MAE indicates the level of similarity between predicted and experimentally measured values;
RMSE represents the overall deviation between predicted and experimentally measured values, and MAPE can be considered as the relative error to the experimentally measured values [42]. The model with higher $R^{2}$ and lower MAE, RMSE, and MAPE can be regarded as having better performance in the prediction.

$R^{2}=\frac{\left(n \sum y_{i} y_{i}^{\prime}-\sum y_{i} \sum y_{i}^{\prime}\right)^{2}}{\left(n \sum y_{i}^{2}-\sum y_{i}^{2}\right)\left(n \sum y_{i}^{\prime 2}-\left(\sum y_{i}^{\prime}\right)^{2}\right)}$

$M A E=\frac{1}{n} \sum_{i=1}^{n}\left|y_{i}-y_{i}^{\prime}\right|$

$R M S E=\sqrt{\frac{1}{n} \sum_{i=1}^{n}\left(y_{i}-y_{i}^{\prime}\right)^{2}}$

$M A P E=\frac{100 \%}{n} \sum_{i=1}^{n} \frac{\left|y_{i}-y_{i}^{\prime}\right|}{\left|y_{i}\right|}$

The overall performance of $k$-fold cross-validation was calculated by averaging each of the abovementioned indicators of $k$ individual folds:

$A_{C V}=\frac{\sum_{i}^{k} P_{i}}{k}$

where $A_{C V}$ denotes the averaged value of the performance indicators for the $k$-fold cross-validation, and $P_{i}$ is the value of the abovementioned performance indicator for the $i^{\text {th }}$ fold.

\subsection{Test of the superiority of model performance}

A superiority test was carried out to verify if the proposed model performs significantly different from the other models. As a common method for multiple model comparison,
Fig. 3 The structure of ANN models: (a) mass change prediction; (b) compressive strength prediction
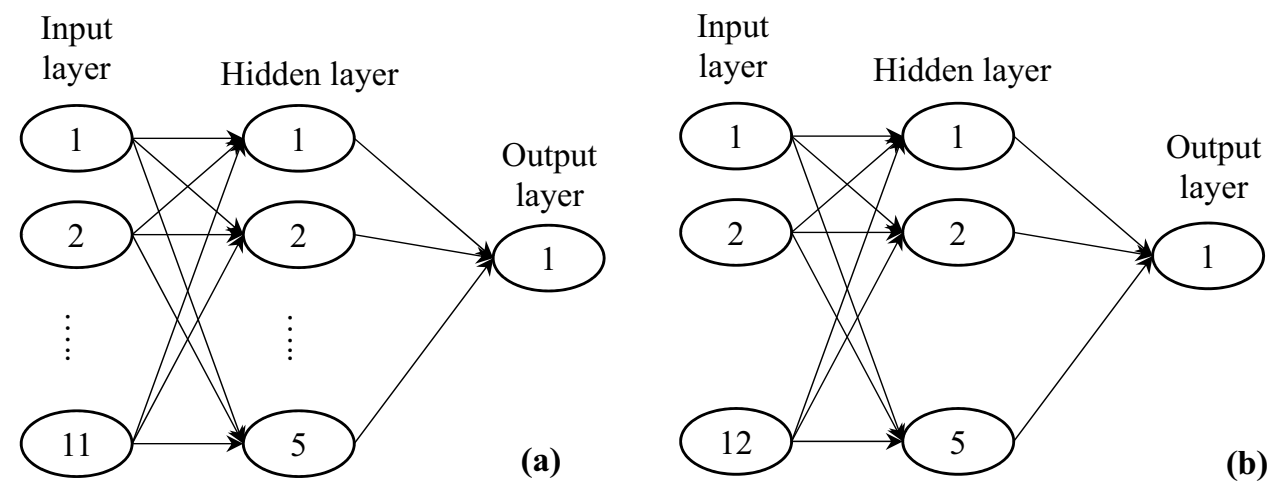
Friedman's test method was adopted in this study for the comparison between the six models [31]. Friedman's test is a nonparametric method with a null hypothesis: the mean of forecasting errors are the same for all models [54]. If the null hypothesis is rejected, it means the compared models perform significantly differently. To test this null hypothesis, the test statistic FM is calculated by Eq. (12) [55]. If the calculated FM is larger than the critical FM, it suggests that the null hypothesis of Friedman's test is rejected. In this study, Friedman's test was carried out on the Matlab 2019a platform. The $F M$ values will be calculated along with the $p$-value. The $p$-value is the probability of critical $F M>$ calculated $F M$. A low $p$-value suggests that the null hypothesis of Friedman's test is rejected, indicating a significant difference between compared models.

$F M=\left[\frac{12}{N_{F} \times k_{F} \times\left(k_{F}+1\right)}\right] \times \sum R_{F}^{2}-\left[3 \times N_{F} \times\left(k_{F}+1\right)\right]$

where $N_{F}$ is the number of parameters used for performance evaluation; $k_{F}$ is the number of models for comparison; $R_{F}$ is the sum of the average rank of performance parameters for each model, which can be calculated by Eq. (13) [55].

$R_{F}=\frac{1}{N_{F}} \sum_{i=1}^{N_{F}} s_{i}$

where $s_{i}$ is the rank of the ith parameter for performance evaluation for each model.

\section{Development of database}

To predict the deterioration of mortar samples under sulfuric acid attack, data used in this study were obtained from experimental work on 19 mixtures. The input parameters include both mixture design and immersion conditions. These immersion conditions are: the $\mathrm{pH}$ of immersion acid, wetting-drying cycle, immersion ages, number of times acid was added, and ratios of acid volume to sample surface area. The mixture design parameters are: fly ash content, silica fume content, nano-silica content, cellulose nanocrystal content, calcium aluminate cement content, and geopolymers. The monitoring of deterioration of samples during immersion involves measuring the mass change and the residual compressive strength. In this study, two Bayesian-SVR models were constructed. Model I was constructed to predict mass change by considering the abovementioned 11 input parameters ( 6 about mixture design, and 5 about testing conditions), while Model II was constructed to forecast the compressive strength, taking 12 input parameters into account (those parameters from Model I and the compressive strength after 28 days of curing). A total of 93 data points was collected for the prediction of compressive strength and 244 data points were collected for the prediction of mass change. Detailed experimental work is described below.

\subsection{Laboratory experimental work}

In this study, both geopolymer mortars and ordinary Portland cement (OPC) mortars were cast for sulfuric acid immersion test. For OPC mortars, a water-to-cement ratio of 0.45 was used for the mixture design. Admixtures were added at various dosages, as shown in Table 1. The readily mixed mixtures were cast into $\Phi 50 \mathrm{~mm} \times 100 \mathrm{~mm}$ cylinder molds, and then de-molded and transferred at $24 \mathrm{~h}$ to a standard curing room (with a temperature of $25 \pm 2{ }^{\circ} \mathrm{C}$ and relative humidity of $100 \%$ ) for 28 days. For geopolymer mortars, metakaolin was used as the raw material, a mixture of $\mathrm{NaOH}$ solution and $45 \% \mathrm{Na}_{2} \mathrm{SiO}_{3}$ solution was used as the activator, as shown in Table 2 . The readily mixed mixtures were cast into $\Phi 50 \times 100 \mathrm{~mm}$ cylinder molds. In order that the geopolymerization process is accelerated, the cylinder molds were then stored in an oven at a temperature of $40{ }^{\circ} \mathrm{C}$ [56]. After $24 \mathrm{~h}$, the samples were de-molded. In order to avoid moisture loss and surface carbonization, the de-molded samples were then transferred to a sealed plastic container [57]. The sealed container was stored in a standard curing room (with a temperature of $25 \pm 2{ }^{\circ} \mathrm{C}$ and relative humidity of $100 \%$ ) until 28 days.

After 28 days of standard curing, samples were immersed in sulfuric acid with $\mathrm{pH}$ values of $1.5,2,3,4.5$, and 6.5. The ratios of acid volume to sample surface area were set to $0.566,0.453,0.377,0.262,0.203$, and 0.119 . To keep the acidity of the immersion acid, the concentrated sulfuric acid was added to the immersing solution frequently. The number of times the acid was added was also recorded. The immersion tests were terminated after three months or 75 days of immersion. The residual compressive strength was monitored per ASTM C42-16 [58] on 7, 21, $42,56,75,88$, and 91 days of immersion. The weight of samples was measured at intervals of 7 or 15 days using an electronic scale with an accuracy of $\pm 0.1 \mathrm{~g}$. Before the weighing, samples were dried under room temperature and re-immersed in sulfuric acid after weighing (wettingdrying cycle). The mass change was calculated in the following equation:

$$
\Delta m=\frac{m_{n}-m_{0}}{m_{0}} \times 100 \%
$$


Table 1 Mix proportion of OPC-based mortars

\begin{tabular}{lllllll}
\hline Mixtures & Cement $\left(\mathrm{kg} / \mathrm{m}^{3}\right)$ & Water $\left(\mathrm{kg} / \mathrm{m}^{3}\right)$ & Sand $\left(\mathrm{kg} / \mathrm{m}^{3}\right)$ & $\begin{array}{l}\text { Admixture } \\
\left(\mathrm{kg} / \mathrm{m}^{3}\right)\end{array}$ & Admixture type & $\begin{array}{l}\text { Admixture } \\
\text { content, } \%\end{array}$ \\
\hline Reference & 493.25 & 221.96 & 1541.4 & 0.00 & N/A & 0 \\
FA-5 & 468.59 & 221.96 & 1541.4 & 24.66 & Fly ash & 0.45 \\
FA-10 & 443.92 & 221.96 & 1541.4 & 49.32 & Fly ash & 0.45 \\
FA-20 & 394.60 & 221.96 & 1541.4 & 98.65 & Fly ash & 10 \\
FA-30 & 345.27 & 221.96 & 1541.4 & 147.97 & Fly ash & 20 \\
SF5 & 468.59 & 221.96 & 1541.4 & 24.66 & Silica fume & 30 \\
SF10 & 443.93 & 221.96 & 1541.4 & 49.33 & Silica fume & 5 \\
SF15 & 419.26 & 221.96 & 1541.4 & 73.99 & Silica fume & 0.45 \\
NS0.5 & 490.78 & 221.96 & 1541.4 & 2.47 & Nano-silica & 0.45 \\
NS1.0 & 488.32 & 221.96 & 1541.4 & 4.93 & Nano-silica & 0.45 \\
NS1.5 & 485.85 & 221.96 & 1541.4 & 7.41 & Nano-silica & 0.45 \\
NS2.0 & 483.39 & 221.96 & 1541.4 & 9.88 & Nano-silica & 1.0 \\
C-0.2 & 493.25 & 221.96 & 1541.4 & 0.49 & Cellulose nanocrystal & 0.2 \\
C-0.4 & 493.25 & 221.96 & 1541.4 & 0.97 & Cellulose nanocrystal & 0.4 \\
C-1 & 493.25 & 221.96 & 1541.4 & 2.43 & Cellulose nanocrystal & 1.0 \\
C-1.5 & 493.25 & 221.96 & 1541.4 & 3.64 & Cellulosenanocrystal & 1.5 \\
\hline
\end{tabular}

Table 2 Mix proportions of metakaolin-based geopolymer mortars

\begin{tabular}{|c|c|c|c|c|c|c|}
\hline \multirow[t]{2}{*}{ Mixture ID } & \multicolumn{2}{|c|}{ Raw materials, $\mathrm{g}$} & \multirow[t]{2}{*}{ Sand, $\mathrm{g}$} & \multicolumn{3}{|c|}{ Alkali activator, $\mathrm{g}$} \\
\hline & Metakaolin & $\begin{array}{l}\text { Calcium alumi- } \\
\text { nate cement }\end{array}$ & & $\mathrm{NaOH}$ & $\mathrm{Na}_{2} \mathrm{SiO}_{3}$ & Water \\
\hline GM & 504 & 0 & 1512 & 66.1 & 179.2 & 374.36 \\
\hline MC5 & 478.8 & 25.2 & 1512 & 66.1 & 179.2 & 374.36 \\
\hline MC10 & 453.6 & 50.4 & 1512 & 66.1 & 179.2 & 374.36 \\
\hline
\end{tabular}

where $\Delta m$ is the mass change in percent, $m_{0}$ is the mass of the sample before immersion, and $m_{n}$ is the mass of the sample after a certain period of immersion.

\subsection{Data preprocessing}

The values of different input parameters may vary drastically in magnitude; this could affect the accuracy and efficiency of the model significantly because the parameters with a greater range may dominate the training process compared with parameters with a smaller range [38]. Thus, the data collected for SVR modeling are normalized to [0, 1] according to the following equation:

$x^{\prime}=\frac{x-x_{\min }}{x_{\max }-x_{\min }}$

where $x^{\prime}$ is the standardized data, $x_{\min }$ is the minimum value of the parameter, and $x_{\max }$ is the maximum value of the parameter.

\subsection{Grey relational analysis}

Since the selection of the input parameters has a great impact on the accuracy of the prediction performance, how close the selected input parameters are to the output parameters needs to be examined. In this study, the grey relational analysis was carried out after data normalization to verify if the selected input parameters have a reasonably close relation with the output parameters. The grey relational analysis measures the closeness between one parameter and all the other parameters [59]. In the grey relational analysis, the relevancy between two parameters is measured by the grey relational grade. The details for the calculation of the grey relational grades can be referred to the study by Chan et al. [60] and You et al. [61]. A higher grade represents that the input parameter is more closely related to the output parameter [62].

The calculated grey relational grades are listed in Tables 3 and 4 for mass change prediction and compressive strength prediction, respectively. A higher grey relational grade (closer to 1 ) reflects a greater influence of 
Table 3 Grey relational grades between input factors with mass change

\begin{tabular}{ll}
\hline Input parameters & $\begin{array}{l}\text { Grey relational } \\
\text { grades to mass } \\
\text { change }\end{array}$ \\
\hline Geopolymer & 0.6892 \\
CAC content, \% & 0.6901 \\
CNC content, \% & 0.6864 \\
Fly ash content, \% & 0.6896 \\
Silica fume content, \% & 0.6743 \\
Nano-silica content, \% & 0.6870 \\
Wet-dry cycle & 0.6050 \\
Ratio of sample surface to acid volume & 0.6880 \\
pH & 0.6143 \\
Time, day & 0.2519 \\
Acid addition times & 0.4863 \\
\hline
\end{tabular}

Table 4 Grey relational grades between input factors with compressive strength

\begin{tabular}{ll}
\hline Input parameters & $\begin{array}{l}\text { Grey relational grades } \\
\text { to compressive } \\
\text { strength }\end{array}$ \\
\hline Geopolymer & 0.4356 \\
CAC content, \% & 0.4404 \\
CNC content, \% & 0.4354 \\
Fly ash content, \% & 0.4407 \\
Silica fume content, \% & 0.4431 \\
Nano-silica content, \% & 0.4358 \\
Wet-dry cycle & 0.7761 \\
Ratio of sample surface to acid volume & 0.4424 \\
pH & 0.5502 \\
Time, day & 0.1709 \\
Acid addition times & 0.5740 \\
Initial compressive strength & 0.3082 \\
\hline
\end{tabular}

the input parameter on the output parameter. It can be seen that the selected input parameters have a reasonably close relationship with the mass change and compressive strength. The grades ranged from 0.252 to 0.690 for inputs of mass change prediction and from 0.171 to 0.776 for inputs of compressive strength prediction.

\section{Results and discussion}

To assess the performance of the Bayesian optimization technique in searching for the optimal hyperparameters, SVR models with three other optimization techniques (GS, RS and PSO) were also constructed for comparison in this study. Then, the SVR models were compared with the currently popular TSVR and traditional ANN models.
The five-fold cross-validation technique was applied to all six models. The performance of these models was evaluated and compared using the parameters in Sect. 2.4 (i.e., $\left.R^{2}, \mathrm{MAE}, \mathrm{RMSE}, \mathrm{MAPE}\right)$. The above predictive models were independently applied in predicting mass change and compressive strength.

\subsection{Prediction results of mass change}

The mass change was predicted using six predictive models for mortars soaked in sulfuric acid under various immersion conditions. Eleven input parameters were incorporated in the models, including 6 mixture design parameters and 5 parameters about immersion conditions. The five-fold cross-validation technique was applied to test the accuracy of the model. The total of 244 data points was divided into five parts, with the sizes of each part being $(48,49,49,49,49)$. One part of the data was used for testing the models, while the other four parts were fed to the models for the training process. For SVR models, the training process is to find the optimal hyperparameters $(\epsilon, C, \sigma)$. To compare the optimization performance of the four different techniques, Fig. 4 illustrates the hyperparameter optimization process of each technique (data part 5 as testing data). The objective function (MSE) decreased with the increasing iteration number in all the optimization techniques. All the optimization processes converged before the set maximum iteration number. The objective function converged at iterations of around 50 with a value of 0.0454 for the $\mathrm{BO}$ technique, indicating an excellent efficiency in searching for the optimal hyperparameter for the SVR model. This is because the BO technique makes full use of information from previous iterations to find the next possible combination of hyperparameters [41, 42]. The results of the objective function and hypermeters after optimization (training process) are also listed in Table 5. The BO technique was able to find the hypermeters combination with the lowest objective function value, compared with other techniques. In short, the BO technique outperformed the other three techniques of SVR model optimization.

To compare the performance of BO-SVR with the five other predictive models in forecasting mass change, the five-fold average $R^{2}, \mathrm{MAE}, \mathrm{RMSE}$, and MAPE were calculated for each predictive model from the test data sets; the results are listed in Table 6 along with the running time. Compared with other optimization techniques, the $\mathrm{BO}$ technique required relatively short running time. The $\mathrm{BO}-$ SVR had a running time of $1330 \mathrm{~s}$, while the GS-SVR and PSO-SVR needed $4550 \mathrm{~s}$ and $12578 \mathrm{~s}$, respectively. However, compared with the traditional ANN and the TSVR, the BO-SVR did not show improvement in running time (769 s for TSVR, $15.82 \mathrm{~s}$ for ANN). 

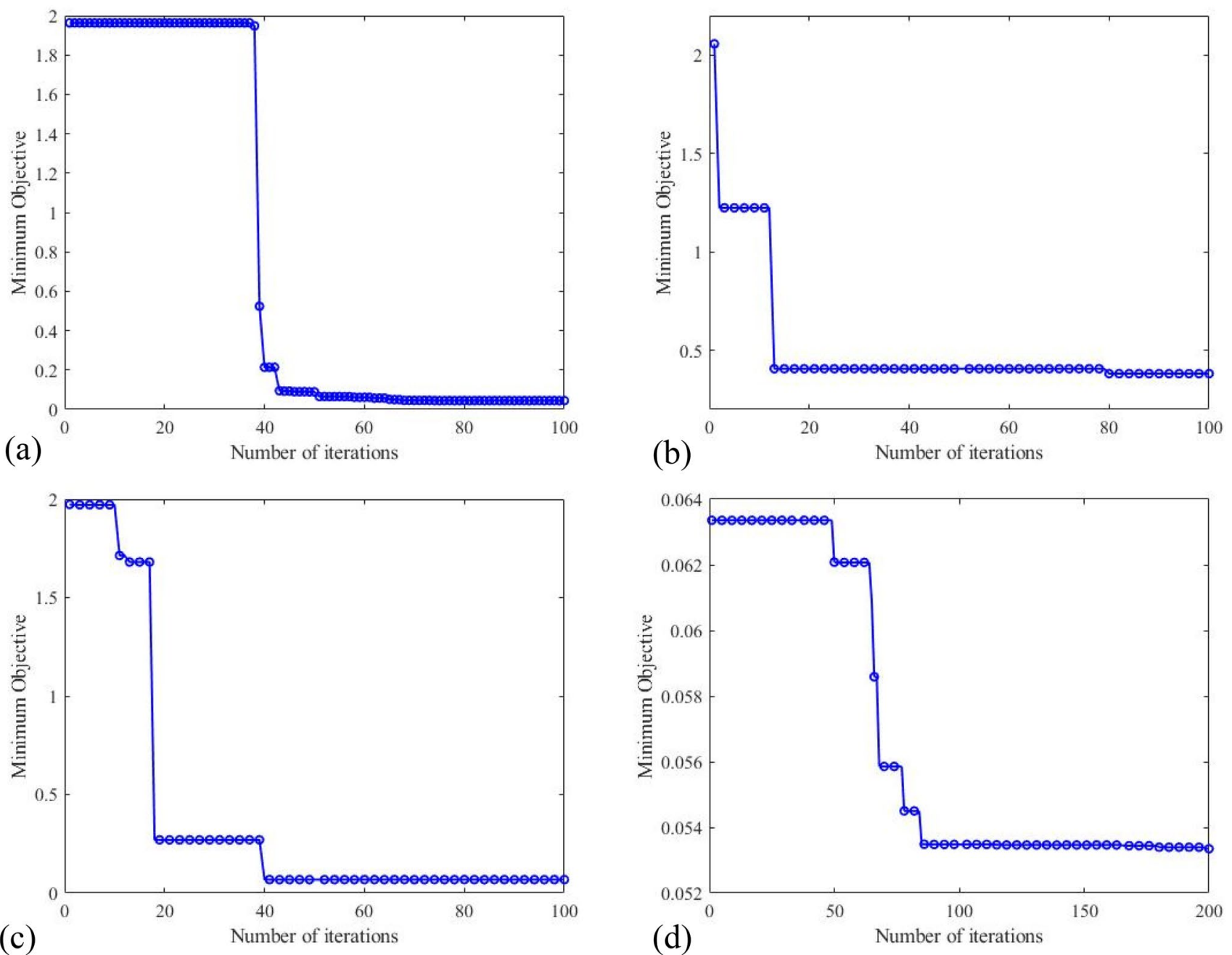

Fig. 4 Optimization process in mass change prediction (data part 5 as testing data): (a) BO-SVR; (b) GS-SVR; (c) RS-SVR; (d) PSO-SVR

Table 5 Optimization results of each technique (data part 5 as testing data)

\begin{tabular}{lll}
\hline $\begin{array}{l}\text { Optimization } \\
\text { technique }\end{array}$ & $\begin{array}{l}\text { Objective function } \\
\text { value }\end{array}$ & $(\epsilon, C, \sigma)$ \\
\hline BO & 0.0454 & $(0.0017,202.6242,4.4972)$ \\
GS & 0.3815 & $(0.0086,46.4159,2.1544)$ \\
RS & 0.0679 & $(0.0059,870.0991,4.2942)$ \\
PSO & 0.0530 & $(0.0913,812.6573,5.6815)$ \\
\hline
\end{tabular}

Regarding the accuracy, it can be seen from Table 6 that all of the models were able to predict the mass change with high accuracy. The minimum $R^{2}$ was obtained with the TSVR model having an $R^{2}$ of 0.8483 . It can be seen from MAE and RMSE results that the performance of these models in mass change prediction was found in the order of BO-SVR $>$ RS-SVR $>$ ANN $>$ GS-SVR $>$ PSO-SVR $>$ TSVR. The ANN model showed an intermediate level of accuracy among the six models. It produced a prediction with $R^{2}$ of 0.9886 , MAE of $0.1621 \%$, and RMSE of $0.1200 \%$. The highest accuracy was achieved with the BO-SVR model, with an $R^{2}$ of 0.9914 . The BO-SVR was able to predict the mass change with small errors (MAE of $0.1471 \%$, RMSE of $0.0804 \%$ ). The MAE of the BO-SVR was almost 5 times lower than that of TSVR. The excellent accuracy in predicting mass change is visually illustrated in Fig. 5 for the BO-SVR model. For each experimentally measured mass change, there is a predicted value that is closely matching. However, the values of MAPE, which can be considered as relative errors to measured data, are noticeably large for all models, with values of $50.79 \%, 58.52 \%, 49.93 \%$, $69.92 \%$, and $44.30 \%$ for BO-SVR, GS-SVR, RS-SVR, PSOSVR, and ANN, respectively. This is because, obtained from the experimental study, a large portion of the data points (mass change) was close to zero. The MAPE values would be significantly amplified as the output approaches zero 
Table 6 Performance evaluation of different predictive models

\begin{tabular}{llllll}
\hline $\begin{array}{l}\text { Predictive } \\
\text { techniques }\end{array}$ & Average $R^{2}$ & Average MAE, \% & Average RMSE, \% & Average MAPE, \% & Running time, $\mathrm{s}$ \\
\hline BO-SVR & 0.9914 & 0.1471 & 0.0804 & 50.79 & 1330.66 \\
GS-SVR & 0.9771 & 0.1841 & 0.1433 & 58.52 & 4450.33 \\
RS-SVR & 0.9868 & 0.1545 & 0.0835 & 49.93 & 622.96 \\
PSO-SVR & 0.9841 & 0.1855 & 0.1223 & 69.92 & 12578.11 \\
TSVR & 0.8483 & 0.676 & 0.9759 & 278.83 & 769.78 \\
ANN & 0.9886 & 0.1621 & 0.1200 & 44.30 & 15.82 \\
\hline
\end{tabular}

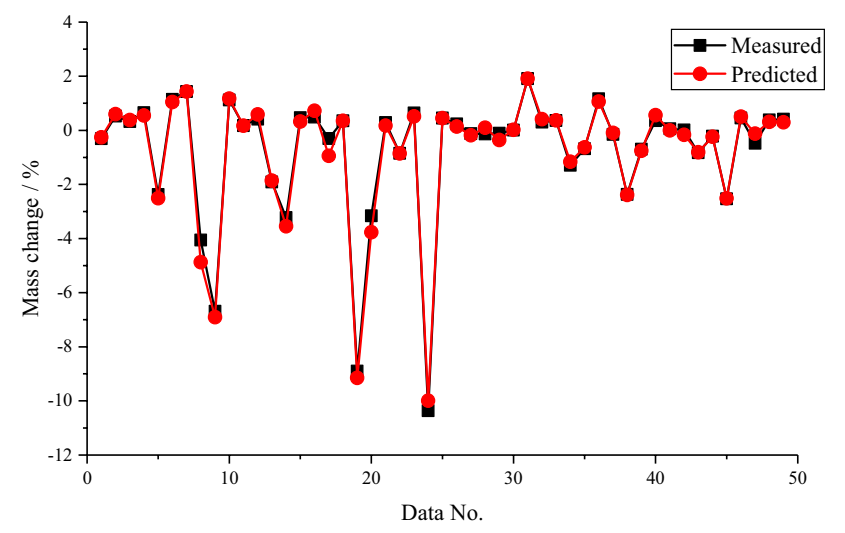

Fig. 5 BO-SVR forecasting results of testing data part 5

[63]. Thus, the MAPE was not considered the main evaluation parameter for mass change prediction.

A Friedman's test was also conducted to examine the superiority of BO-SVR model over the other five models. The calculated FM value was 10.67, with a $p$-value of 0.015 . At the significance level of 0.05 , as the $p$-value is lower than 0.05 , it is concluded that there is a significant difference between BO-SVR and the other five models. Since the BO-SVR had the lowest errors, this significant difference suggests that the BO-SVR model performed considerably better than the other five models in predicting the mass change.

\subsection{Prediction results of compressive strength}

For the prediction of residual compressive strength after immersion, twelve input parameters were included (i.e., those parameters in mass change prediction and compressive strength on the 28th day of curing). A total of 93 data points were used and divided into five parts with the sizes of $(18,19,19,19,18)$ in the modeling. During the training process of SVR models, the optimal hyperparameters combinations were obtained by BO, GS, RS, and PSO techniques. The evolutions of the objective function (e.g., MSE) with iterations were plotted in Fig. 6 for all SVR models. The optimization results of the training process are presented in Table 7. It can be seen from Table 7 that all the models converged before reaching the maximum iteration number. The BO and GS converged with relatively fewer iteration steps-about 20 iterations for $\mathrm{BO}$ and 5 iterations for RS. The GS and PSO found their optimal solution at iterations of 60 and 155, respectively. Compared with the other techniques, the $\mathrm{BO}$ technique obtained a hyperparameters combination of $(0.7969,299.85683 .2164)$ with a low objective function value of 2.5161 (see Table 7).

To assess the performance of each model, the averaged $R^{2}, \mathrm{MAE}, \mathrm{RMSE}$, and MAPE were calculated. The results are listed in Table 8 , along with the running time. Among these models, BO-SVR showed a relatively short running time of $961 \mathrm{~s}$. The lowest accuracy was found with the ANN, with $R^{2}$ of 0.6691, MAE of $2.8005 \mathrm{MPa}$, RMSE of $14.3192 \mathrm{MPa}$, and MAPE of $9.46 \%$. Following the ANN model, the TSVR showed comparable accuracy in compressive strength prediction $\left(R^{2}\right.$ of $0.7021, \mathrm{MAE}$ of 2.4069 MPa, RMSE of $15.2562 \mathrm{MPa}$, and MAPE of $7.5155 \%$ ). The BO-SVR demonstrated the best performance among the six predictive models. It showed a high $R^{2}$ of 0.8720 and only $5.7423 \%$ of MAPE. The BO-SVR forecasting results of data part 5 are also presented in Fig. 7 to visually show the high accuracy in compressive strength prediction. The predicted values are closely matched with the experimentally measured values. In addition, it is noted that the prediction accuracy was ranked as BO-SVR $>$ RS-SVR $>$ GS-SVR $>$ PSO-SVR $>$ TSVR $>$ ANN.

To ensure that the improvement in predicting accuracy is significant by the proposed BO-SVR model, Friedman's test was carried to investigate the superiority of BO-SVR over the other five models in predicting the compressive strength. A $p$-value of 0.0013 was obtained, which indicates a significantly better performance of BO-SVR over the other five models.

\subsection{Evaluation of the proposed model}

The proposed BO-SVR model was able to predict the mass change and compressive strength of mortar samples with high accuracy. Among the four optimization techniques, the Bayesian algorithm outperformed the 

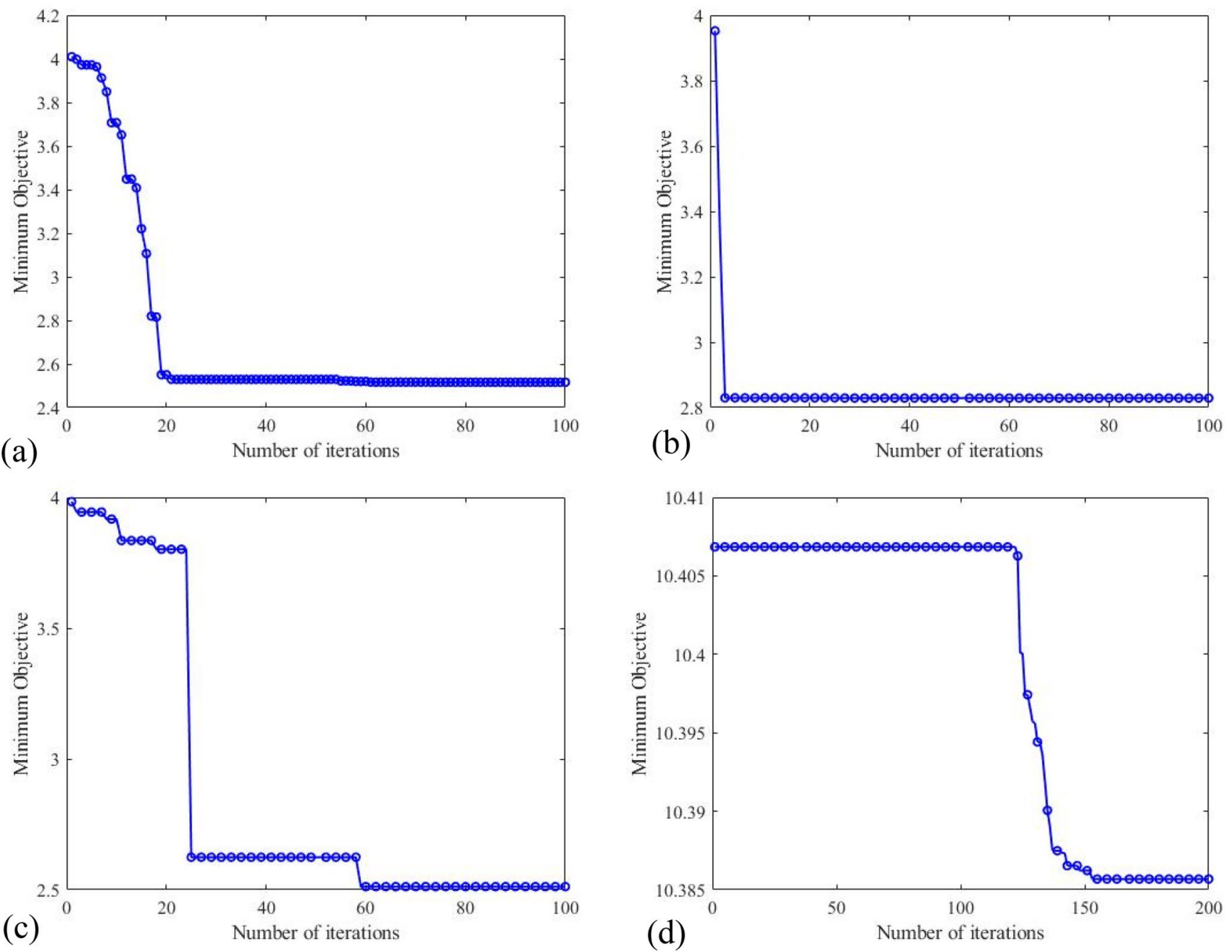

Fig. 6 Optimization process in compressive strength prediction (data part 5 as testing data): (a) BO-SVR; (b) GS-SVR; (c) RS-SVR; (d) PSO-SVR

Table 7 Optimization results of each technique (data part 5 as testing data)

\begin{tabular}{lll}
\hline $\begin{array}{l}\text { Optimization } \\
\text { technique }\end{array}$ & $\begin{array}{l}\text { Objective function } \\
\text { value }\end{array}$ & $(\epsilon, \mathrm{C}, \sigma)$ \\
\hline BO & 2.5161 & $(0.7969,299.85683 .2164)$ \\
GS & 2.8290 & $(0.0308,10,2.1544)$ \\
RS & 2.5119 & $(0.0547,362.3195,2.3748)$ \\
PSO & 10.39 & $(0.2557,82.2266,3.7792)$ \\
\hline
\end{tabular}

other three in both mass change prediction and compressive strength prediction. The Bayesian algorithm was able to find the best hyperparameters combination for SVR models that can result in the minimum value of the objective function. For example, a minimum value of the objective function of 0.0454 was achieved during the training process by the $\mathrm{BO}$ technique for mass change prediction, while the values of objective function were
$0.3815,0.0679$, and 0.0530 for GS, RS, and PSO, respectively. Compared with the most commonly used ANN model, the Bayesian-optimized SVR provided a much accurate prediction on mass change and compressive strength of sulfuric acid immersed samples. For instance, the BO-SVR-predicted compressive strength had a $k$-fold averaged MAPE of $5.7423 \%$, which is much smaller than 8.4614\% from the ANN model.

Despite its excellent performance, the proposed BOSVR model has its limitations in this study. Much work will be required to further the model. For example, although this model has considered many testing conditions as the input parameters (e.g., the $\mathrm{pH}$ of immersion acid, wetting-drying cycle, immersion ages, number of times of the acid addition, and ratios of acid volume to sample surface area), there are more conditions to incorporate. For instance, some studies accelerated the deterioration rate by brushing the samples [7, 64], while sample brushing was not included as an input 
Table 8 Performance evaluation of different predictive models

\begin{tabular}{llllll}
\hline $\begin{array}{l}\text { Predictive } \\
\text { techniques }\end{array}$ & Average $R^{2}$ & $\begin{array}{l}\text { Average } \\
\text { MAE, MPa }\end{array}$ & Average RMSE, MPa & Average MAPE, \% & Running time, $s$ \\
\hline BO-SVR & 0.8720 & 1.7659 & 6.9268 & 5.7423 & 961.65 \\
GS-SVR & 0.7924 & 2.3798 & 11.8631 & 7.6802 & 1029.66 \\
RS-SVR & 0.8048 & 2.2108 & 10.5025 & 7.4465 & 111.56 \\
PSO-SVR & 0.7614 & 2.4069 & 15.2562 & 7.5155 & 1515.10 \\
TSVR & 0.7021 & 2.8005 & 14.3192 & 9.46 & 302.59 \\
ANN & 0.6691 & 2.4614 & 19.8146 & 8.4614 & 17.91 \\
\hline
\end{tabular}

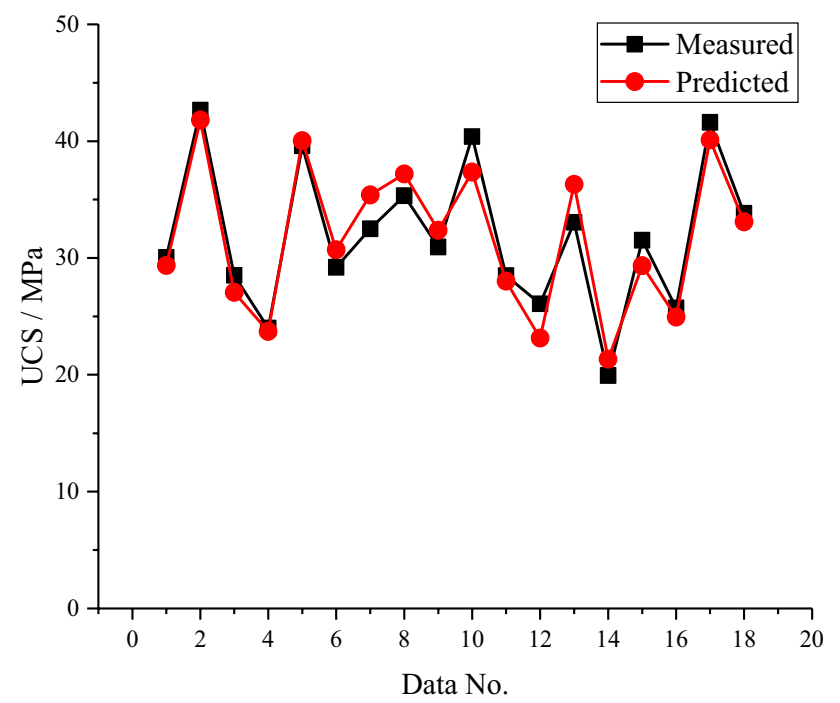

Fig. 7 BO-SVR forecasting results of testing data part 5

parameter in this model. Thus, more parameters, such as sample brushing and also immersion temperature [65], should be included in the model to better mimic the real conditions.

\section{Conclusion}

The objective of this study was to construct a support vector regression model with Bayesian optimization (BO-SVR) to predict the mass change and compressive strength of mortar samples under a sulfuric acid attack. The support vector regression model with Bayesian optimization was compared with five other predictive models. These models include grid search-support vector regression (GS-SVR), random search-support vector regression (RS-SVR), particle swarm optimization-support vector regression (PSO-SVR), twin SVR and traditional artificial neural network (ANN). Five parameters of testing conditions and six mixture design parameters were used as input parameters. In total, 93 data points were obtained for compressive strength prediction, and
244 data points were obtained for mass change prediction. The $k$-fold cross-validation technique was adopted to test the accuracy of the models. The performance of each model was evaluated by four parameters: the coefficient of determination $R^{2}$, the mean absolute error, the root mean square error and the mean absolute percent error. It was found that the proposed BO-SVR model outperformed the other five models and provided high accuracy in predicting the mass change and the compressive strength of mortar samples under the sulfuric acid attack. Compared with other optimization techniques, the Bayesian optimization was efficient (with relatively fewer iterations) and provided better solutions in searching for the optimal hypermeters for the SVR model. Despite its excellent performance, the proposed BO-SVR model needs to include more input parameters about mixture design and testing conditions to better mimic real conditions.

Acknowledgement The authors are thankful for the financial support from the Natural Sciences and Engineering Research Council (NSERC) -EGP 501335-16 and RGPIN-2017- 05537, Canada.

\section{Compliance with ethical standards}

Conflict of interest There are no conflicts of interest to disclose.

\section{References}

1. Wu L, Hu C, Liu WV (2019) Effects of pozzolans on acid resistance of shotcrete for sewer tunnel rehabilitation. J Sustainable Cement-Based Mater 8(1):55-77. https://doi.org/10.1080/21650 373.2018.1519645

2. Torii K, Kawamura M (1994) Effects of fly ash and silica fume on the resistance of mortar to sulfuric acid and sulfate attack. Cem Concr Res 24(2):361-370. https://doi.org/10.1016/00088846(94)90063-9

3. Dinakar P, Babu KG, Santhanam M (2008) Durability properties of high volume fly ash self compacting concretes. Cem Concr Compos 30(10):880-886. https://doi.org/10.1016/j.cemconcomp .2008.06.011

4. Ekolu SO, Diop S, Azene F, Mkhize N (2016) Disintegration of concrete construction induced by acid mine drainage attack. J 
S Afr Inst Civ Eng 58(1):34-42. https://doi.org/10.17159/2309$8775 / 2016 / v 58 n 1 a 4$

5. Shetti AP, Das BB (2015) Acid, alkali and chloride resistance of early age cured silica fume concrete. In: Advances in structural engineering: materials, vol 3. Springer India, pp 1849-1862. https://doi.org/10.1007/978-81-322-2187-6_142

6. Reiterman P, Tomek J (2016) Resistance of concrete with Metakaolin addition to acid environment. Key Eng Mater 677:144-149. https://doi.org/10.4028/www.scientific.net/KEM.677.144

7. De Belie N, Monteny J, Beeldens A, Vincke E, Van Gemert D, Verstraete W (2004) Experimental research and prediction of the effect of chemical and biogenic sulfuric acid on different types of commercially produced concrete sewer pipes. Cem Concr Res 34(12):2223-2236. https://doi.org/10.1016/j.cemco nres.2004.02.015

8. Kurtis KE, Monteiro PJ, Madanat SM (2000) Empirical models to predict concrete expansion caused by sulfate attack. ACI Mater J 97(2):156-161

9. Marchand J, Odler I, Skalny J (2002) Sulfate attack on concrete. CRC Press, London. https://doi.org/10.4324/9780203301623

10. Lee K-M, Bae S-H, Park J-I, Kwon S-O (2015) Mass change prediction model of concrete subjected to sulfate attack. Math Probl Eng 2015:298918

11. Hewayde E, Nehdi M, Allouche E, Nakhla G (2007) Neural network prediction of concrete degradation by sulphuric acid attack. Struct Infrastruct Eng 3(1):17-27

12. Alani AM, Faramarzi A (2014) An evolutionary approach to modelling concrete degradation due to sulphuric acid attack. Appl Soft Comput 24:985-993

13. Rombén $L$ (1980) Aspects on testing methods for acid attacks on concrete-further experiments, vol 12. Swedish Cement and Concrete Research Institute, Sweden

14. Wafa F (1994) Accelerated sulfate attack on concrete in a hot climate. Cem Concr Aggregates 16(1):31-35

15. Attiogbe EK, Rizkalla SH (1988) Response of concrete to sulfuric acid attack. ACI Mater J 85(6):481-488

16. Chen H, Qian C, Liang C, Kang WJP (2018) An approach for predicting the compressive strength of cement-based materials exposed to sulfate attack. PLoS One 13(1):e0191370

17. Kaviani S, Sohn I (2020) Influence of random topology in artificial neural networks: a survey. ICT Express. https://doi.org/10.1016/j. icte.2020.01.002

18. Xin R, Zhang J, Shao Y (2020) Complex network classification with convolutional neural network. Tsinghua Sci Technol 25(4):447-457. https://doi.org/10.26599/TST.2019.9010055

19. Panchal G, Ganatra A, Shah P, Panchal D (2011) Determination of over-learning and over-fitting problem in back propagation neural network. Int J Soft Comput 2(2):40-51

20. Lawrence S, Giles CL, Tsoi AC (1997) Lessons in neural network training: overfitting may be harder than expected. In: AAAI/IAAI. Citeseer, pp 540-545

21. Wellmann R, Harmand P, Müller CH (2009) Distribution-free tests for polynomial regression based on simplicial depth. J Multivar Anal 100(4):622-635. https://doi.org/10.1016/j. jmva.2008.06.009

22. Moraes R, Valiati JF, Gavião Neto WP (2013) Document-level sentiment classification: an empirical comparison between SVM and ANN. Expert Syst Appl 40(2):621-633. https://doi. org/10.1016/j.eswa.2012.07.059

23. Antonanzas J, Urraca R, Pernía-Espinoza A, Aldama A, FernándezJiménez LA, Martínez-de-Pisón FJ (2017) Single and blended models for day-ahead photovoltaic power forecasting. In: International conference on hybrid artificial intelligence systems, Cham. Springer International Publishing, pp 427-434

24. Gao Y, Song Z (2013) Time series prediction model of concrete corrosion in sulfuric based on SVM. In: 2013 international conference on computer sciences and applications, Wuhan, China. IEEE, pp 560-563

25. Kaytez F, Taplamacioglu MC, Cam E, Hardalac F (2015) Forecasting electricity consumption: a comparison of regression analysis, neural networks and least squares support vector machines. Int J Electr Power Energy Syst 67:431-438

26. Pu Y, Apel DB, Wang C, Wilson B (2018) Evaluation of burst liability in kimberlite using support vector machine. Acta Geophys 66(5):973-982

27. Czarnecki WM, Podlewska S, Bojarski AJ (2015) Robust optimization of SVM hyperparameters in the classification of bioactive compounds. J Cheminform 7(1):38

28. Cortes C, Vapnik V (1995) Support-vector networks. J Mach Learn 20(3):273-297

29. Kavaklioglu K (2011) Modeling and prediction of Turkey's electricity consumption using support vector regression. Appl Energy 88(1):368-375. https://doi.org/10.1016/j.apene rgy.2010.07.021

30. Huang Z, Chen H, Hsu C-J, Chen W-H, Wu S (2004) Credit rating analysis with support vector machines and neural networks: a market comparative study. Decis Support Syst 37(4):543-558. https://doi.org/10.1016/S0167-9236(03)00086-1

31. Hong W-C, Dong Y, Lai C-Y, Chen L-Y, Wei S-Y (2011) SVR with hybrid chaotic immune algorithm for seasonal load demand forecasting. Energies 4(6):960-977

32. Laref R, Losson E, Sava A, Siadat M (2019) On the optimization of the support vector machine regression hyperparameters setting for gas sensors array applications. Chemom Intell Lab Syst 184:22-27

33. Smets K, Verdonk B, Jordaan EM (2007) Evaluation of performance measures for SVR hyperparameter selection. In 2007 international joint conference on neural networks, Orlando, Florida, USA, 12-17 Aug. 2007, pp 637-642. https://doi. org/10.1109/IJCNN.2007.4371031

34. Ito K, Nakano R (2003) Optimizing support vector regression hyperparameters based on cross-validation. In: Proceedings of the international joint conference on neural networks, Portland, OR, USA, 20-24 July 2003, pp 2077-2082. https://doi. org/10.1109/IJCNN.2003.1223728

35. Zhang H, Chen L, Qu Y, Zhao G, Guo Z (2014) Support vector regression based on grid-search method for short-term wind power forecasting. J Appl Math 2014:835791. https://doi. org/10.1155/2014/835791

36. Bergstra J, Bengio Y (2012) Random search for hyper-parameter optimization. J Mach Learn Res 13(1):281-305

37. Mantovani RG, Rossi AL, Vanschoren J, Bischl B, De Carvalho AC (2015) Effectiveness of random search in SVM hyper-parameter tuning. In: 2015 international joint conference on neural networks (IJCNN). IEEE, pp 1-8

38. Meng Q, Ma X, Zhou Y (2014) Forecasting of coal seam gas content by using support vector regression based on particle swarm optimization. J Nat Gas Sci Eng 21:71-78

39. Li M, Du W, Nian F (2014) An adaptive particle swarm optimization algorithm based on directed weighted complex network. Math Probl Eng 2014:434972. https://doi. org/10.1155/2014/434972

40. Chou J-S, Chiu C-K, Farfoura M, Al-Taharwa I (2010) Optimizing the prediction accuracy of concrete compressive strength based on a comparison of data-mining techniques. J Comput Civ Eng 25(3):242-253

41. Alade IO, Rahman MAA, Saleh TA (2019) Predicting the specific heat capacity of alumina/ethylene glycol nanofluids using support vector regression model optimized with Bayesian algorithm. Sol Energy 183:74-82 
42. Cheng H, Ding X, Zhou W, Ding R (2019) A hybrid electricity price forecasting model with Bayesian optimization for German energy exchange. Int J Electr Power Energy Syst 110:653-666

43. Peng $X$ (2010) TSVR: an efficient twin support vector machine for regression. Neural Netw 23(3):365-372. https://doi. org/10.1016/j.neunet.2009.07.002

44. Zhong P, Xu Y, Zhao Y (2012) Training twin support vector regression via linear programming. Neural Comput Applic 21(2):399407. https://doi.org/10.1007/s00521-011-0525-6

45. Shao Y-H, Zhang C-H, Yang Z-M, Jing L, Deng N-Y (2013) An $\varepsilon$-twin support vector machine for regression. Neural Comput Applic 23(1):175-185. https://doi.org/10.1007/s0052 1-012-0924-3

46. Ling H, Qian C, Kang W, Liang C, Chen H (2019) Combination of Support Vector Machine and K-Fold cross validation to predict compressive strength of concrete in marine environment. Constr Build Mater 206:355-363. https://doi.org/10.1016/j.conbu ildmat.2019.02.071

47. Hammoudi A, Moussaceb K, Belebchouche C, Dahmoune $F$ (2019) Comparison of artificial neural network (ANN) and response surface methodology (RSM) prediction in compressive strength of recycled concrete aggregates. Constr Build Mater 209:425-436. https://doi.org/10.1016/j.conbuildma t.2019.03.119

48. Behnood A, Golafshani EM (2018) Predicting the compressive strength of silica fume concrete using hybrid artificial neural network with multi-objective grey wolves. J Clean Prod 202:5464. https://doi.org/10.1016/j.jclepro.2018.08.065

49. Kramer MA (1991) Nonlinear principal component analysis using autoassociative neural networks. AIChE J 37(2):233-243

50. Sheela KG, Deepa SN (2013) Review on methods to fix number of hidden neurons in neural networks. Math Probl Eng 2013:425740

51. Doan CD, Liong S-y (2004) Generalization for multilayer neural network bayesian regularization or early stopping. In: Proceedings of Asia Pacific association of hydrology and water resources 2nd conference, pp 5-8

52. Sonebi M, Cevik A, Grünewald S, Walraven J (2016) Modelling the fresh properties of self-compacting concrete using support vector machine approach. Constr Build Mater 106:55-64. https ://doi.org/10.1016/j.conbuildmat.2015.12.035

53. Yaseen ZM, Tran MT, Kim S, Bakhshpoori T, Deo RC (2018) Shear strength prediction of steel fiber reinforced concrete beam using hybrid intelligence models: a new approach. Eng Struct 177:244-255. https://doi.org/10.1016/j.engstruct.2018.09.074

54. López-Vázquez C, Hochsztain E (2019) Extended and updated tables for the Friedman rank test. Commun Stat 48(2):268-281. https://doi.org/10.1080/03610926.2017.1408829
55. Xu J, Shan G, Amei A, Zhao J, Young D, Clark S (2017) A modified Friedman test for randomized complete block designs. Commun Stat 46(2):1508-1519. https://doi.org/10.1080/03610 918.2015.1006777

56. Atiş CD, Görür EB, Karahan O, Bilim C, İlkentapar S, Luga E (2015) Very high strength (120MPa) class $\mathrm{F}$ fly ash geopolymer mortar activated at different $\mathrm{NaOH}$ amount, heat curing temperature and heat curing duration. Constr Build Mater 96:673-678. https ://doi.org/10.1016/j.conbuildmat.2015.08.089

57. Guo X, Shi H, Dick WA (2010) Compressive strength and microstructural characteristics of class $C$ fly ash geopolymer. Cem Concr Compos 32(2):142-147. https://doi.org/10.1016/j.cemco ncomp.2009.11.003

58. ASTM (2016) ASTM C42/C42M-16-standard test method for obtaining and testing drilled cores and sawed beams of concrete. ASTM International, West Conshohocken, PA

59. Sun G, Guan X, Yi X, Zhou Z (2018) Grey relational analysis between hesitant fuzzy sets with applications to pattern recognition. Expert Syst Appl 92:521-532. https://doi.org/10.1016/j. eswa.2017.09.048

60. Chan JWK, Tong TKL (2007) Multi-criteria material selections and end-of-life product strategy: grey relational analysis approach. Mater Des 28(5):1539-1546. https://doi.org/10.1016/j.matde S.2006.02.016

61. You M-L, Shu C-M, Chen W-T, Shyu M-L (2017) Analysis of cardinal grey relational grade and grey entropy on achievement of air pollution reduction by evaluating air quality trend in Japan. J Clean Prod 142:3883-3889. https://doi.org/10.1016/j.jclep ro.2016.10.072

62. Lai W-C, Chang T-P, Wang J-J, Kan C-W, Chen W-W (2012) An evaluation of Mahalanobis distance and grey relational analysis for crack pattern in concrete structures. Comput Mater Sci 65:115-121. https://doi.org/10.1016/j.commatsci.2012.07.002

63. Tayman J, Swanson DA (1999) On the validity of MAPE as a measure of population forecast accuracy. Popul Res Policy Rev 18(4):299-322

64. Monteny J, De Belie N, Taerwe L (2003) Resistance of different types of concrete mixtures to sulfuric acid. Mater Struct 36(4):242-249

65. Mahmoodian M, Alani AM (2017) Effect of temperature and acidity of sulfuric acid on concrete properties. J Mater Civ Eng 29(10):04017154

Publisher's Note Springer Nature remains neutral with regard to jurisdictional claims in published maps and institutional affiliations 